\title{
Modelling of Impedance Matching Circuit with Digital Capacitor in Narrowband Power Line Communication
}

\author{
Chin Pin Rui ${ }^{1}$, Nader Nassif Barsoum ${ }^{2}$, Arthur Wong Kok Ming ${ }^{1}$ and Wong Kiing Ing ${ }^{1}$ \\ 1. Electrical and Computer Engineering Department, Curtin University Sarawak Malaysia, Miri 98009, Sarawak, Malaysia \\ 2. Department of Electrical and Electronic Engineering, University Malaysia Sabah, Kota Kinabalu 44800, Sabah, Malaysia
}

Received: June 09, 2013 / Accepted: August 08, 2013 / Published: January 31, 2014.

\begin{abstract}
Power line communication technology is used in various applications, from high voltage network to the low voltage network, as it is the only wired communication technology that is comparable with wireless communication network. It works by injecting a modulated carrier wave into the electric cables from one transceiver to another. But still, the noise level and impedance mismatch are still the main concern of this technology, particularly in the low voltage network in residential area. Power line has additive non-white noise and extremely harsh environment for communication. At the same time, there is signal attenuation along the power line caused by the impedance mismatch in the power line network. Even though these problems can be controlled using a band-pass filter and an impedance matching circuit respectively, but the impedances in the power line are time and location variant and it is rather difficult to design a circuit that allows maximum power transfer in the system all the time. Thus in this paper, a new adaptive impedance matching circuits is proposed for narrowband power line communication. This methodology is derived based on the RLC band-pass filter circuit. This concept is designed to achieve simpler configuration and higher matching resolution.
\end{abstract}

Key words: Impedance matching, band-pass filter, binary systems, digital capacitor, narrowband power line communication.

\section{Introduction}

As the technology in the world is emerging, wireless communication technology such as infra-red, bluetooth, radio communication, etc. has almost dominating the engineering applications. The main reason is the application of wireless communication technology provides convenience to the user since it does not involve any wiring to perform connection between hardware. However, most of the hardware such as industrial machineries, electrical appliances, automatic gate, security system, etc. still requires electric cable as the power supply in order to operate in correct manner. So in this case, power line communication can be used as another option to perform monitoring and control on the hardware mentioned above.

PLC (power line communication) is a system that

Corresponding author: Chin Pin Rui, B.Eng., M.Phil., research fields: narrowband power line communication, control system and application. E-mail: chinpinrui@ieee.org. transmits information on a conductor which is generally used for electric power transmission $[1,2]$. PLC can be applied at high $(>100 \mathrm{kV})$, medium (1-100 $\mathrm{kV})$ and low $(<1 \mathrm{kV})$ voltage networks. PLC operates by injecting a modulated carrier signal on the wiring network. Different types of PLC use different frequency bands depending on the signal transmission characteristics of the power wiring system [3]. The standard is given according to the CENELEC EN 50065-1. Fig. 1 and Table 1 explain the standard for the power line communication frequency bands in Europe.

There are two classes of PLC system, namely NB-PLC (narrowband power line communication) and BPL (broadband power line) communication. But in this paper, the focus will be on NB-PLC.

PLC is the only wired communication technology that is comparable with wireless communication channel, because it uses the existing power line as the communication medium. However, in LV (low voltage) 


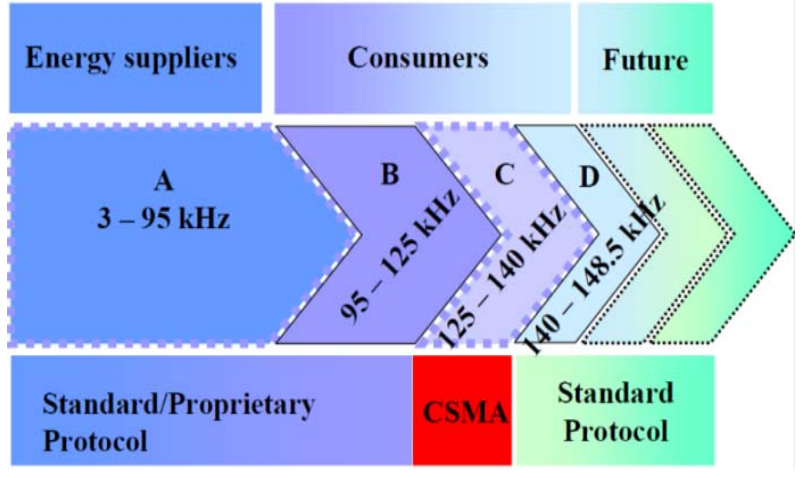

Fig. 1 PLC frequency bands in Europe [4].

Table 1 Frequency range according to CENELEC EN 50065-1.

\begin{tabular}{lcl}
\hline Band & Frequency range & Purpose \\
\hline A & $3-95 \mathrm{kHz}$ & $\begin{array}{l}\text { For electric distribution } \\
\text { companies use and their licenses } \\
\text { Available for consumers with no } \\
\text { restriction } \\
\text { B }\end{array}$ Available for consumers only $^{\text {with media access protocol }}$ \\
C & $125-125 \mathrm{kHz}$ & $\begin{array}{l}\text { Available for consumers with no } \\
\text { restriction }\end{array}$ \\
\hline
\end{tabular}

network, PLC is a simpler network compared to a wireless network, since all the electrical appliances are connected to the power line, through the electrical outlets, for acquiring electrical energy.

The goal of the research presented in this paper is the research of the new methodology of the adaptive impedance matching circuits in narrowband power line communication network that has simpler configuration and higher matching resolution. This paper is organized as follows: Section 1 is the introduction to PLC, and follow by Section 2 is the literature review of NB-PLC, and its problem statement; then Section 3 is the explanation of new impedance matching methodology; after that Section 4 illustrated the simulated verification to verify the feasibility of the concept, and Section 5 concludes the outcome of the research with recommendation for future work.

\section{NB-PLC (Narrowband Power Line Communication)}

NB-PLC technology uses the residential electrical power wiring as a transmission medium to control the lighting and appliances without installation of additional new wiring. Typical home control devices operate by modulating in a carrier wave of between 3 $\mathrm{kHz}$ to $148.5 \mathrm{kHz}$ in the household wiring at the transmitter [5]. The carrier is modulated by digital signals that is generated from the PLC transmitter, and sending it to the receiver. Each receiver in the system has an address and can be individually commanded by the signals transmitted over the household wiring and demodulated at the receiver. These devices may be either plugged into regular power outlets, or permanently wired in place [3].

But then, the PLC application still has problems that need to be improved. For instance, Power line has additive non-white noise and extremely harsh environment for communication $[6,7]$. Particularly in low voltage network, the source of the noise comes from the household machinery and devices and office equipment. The noise will be spread from the electrical appliances into the power line, and therefore it is hard for the signal to transmit along the power line. In general, the power line in the low voltage network has the strong and time-varying non-white noise. Therefore, it is crucial to implement suitable electronic filter to filter out the noise in power line. In this situation, a simple band-pass filter will be good to be used in noise filtering in narrowband power line communication.

Apart of high noise level, impedance mismatch is another major concern of PLC application. The reason is impedance mismatch will leads to data reflection, and therefore the signal attenuated in the power line system [8]. Hence, it is important to perform impedance matching in the PLC systems. However, since impedance in the power line is continuously changing variable depending on time and location, it is rather difficult to match the impedance completely in power line network [5, 9-11]. Thus, the adaptive impedance matching circuit is needed in the PLC in order to achieve ideal maximum power transfer in the systems $[5,9,11]$.

There are a few problems that can be addressed from the current adaptive impedance matching circuit technology. In Ref. [12], the turns of the transformer 
and the value of inductor are altered by sliding the taps, which means it has low resolution of matching unless the number of taps increases. Furthermore, it has high intrinsic parasitic resistance, large size and higher price. Meanwhile for the method used in Ref. [11] requires high numbers of capacitors to achieve high resolution of matching. The better solution was shown in Ref. [9], where VCGIC (voltage controlled general impedance converter) is used. This approach has much higher resolution of matching, but with the limitation that it is not performed well in high current load. Therefore, DIRC was introduced, which can operate in both low and high current load [5]. But this approach requires a high voltage blocking capacitor which has large absolute value of reactance than the inductive reactance in the channel. DIRC (digital inductive reactance converter) bank is needed instead of just a single DIRC to reduce the parasitic resistance of the circuit, which means the circuit becomes more complex.

In this paper, a new approach has been introduced to maximise the matching resolution. A digital capacitor [13] and digital resistor are used to match the source impedance and channel impedance. The impedance in the power line will be measured to provide feedback value to the digital capacitor and digital resistor in order to match the source impedance and channel impedance. Thus, the circuit is simpler, lower noise level and high resolution of matching.

\section{New Impedance Matching Methodology}

This paper proposes a new circuit model for impedance matching using the digital capacitor and digital resistor concept. This circuit model is designed to maximize the matching resolution using simple circuit component.

\subsection{Proposed Impedance Matching Circuit Model}

A simplified circuit diagram of a RLC (resistance-inductance-capacitance circuit) band-pass filter adaptive impedance matching circuit is shown in Fig. 2.

The circuit consists of three parts: The left hand side

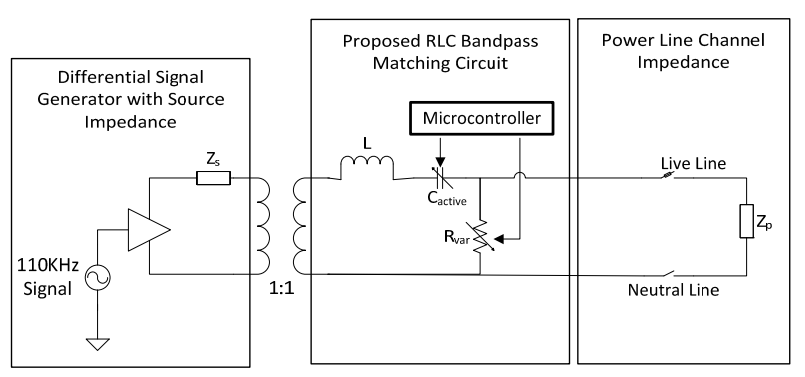

Fig. 2 Proposed RLC band-pass filter adaptive impedance matching circuit.

part is the differential signal generator and the source impedance; the middle part is the proposed RLC band-pass adaptive impedance matching circuit; Meanwhile the right hand side part is the power line channel impedance or load impedance. The impedance matching process occurs when the middle part of the circuit is operating to match both left (source impedance) and right (channel impedance) hand sides of the circuit. This concept works by varying the value of the digital capacitor and digital resistor which controlled by a microcontroller. These values are depending on the feedback value from the measured source impedance and channel impedance.

The RLC band-pass filter circuit model is proposed in this paper as its component can be altered easily, and it can filtered out the noise, allows only the power line modulated frequency to pass through the communication channel.

\subsection{Digital Capacitor}

The digital capacitor is used as the variable capacitor that can be controlled through microcontroller. This concept is proposed in Ref. [13]. The digital capacitor works using binary systems, therefore higher bits yields to higher resolution. Fig. 3 illustrates the example of the digital capacitor, which is 4 bits system, ranging from $1 \mathrm{nF}$ to $15 \mathrm{nF}$. For instance, in order to achieve the value of $12 \mathrm{nF}$, the value 12 in decimal need to be converted into binary, as shown in Eq. (1):

$$
12_{10}=1100_{2}
$$

Then from $1100_{2}$ binary number, the switches 5 and 6 will turn on, and then the capacitive value of 12 $\mathrm{nF}$ will be shown: 


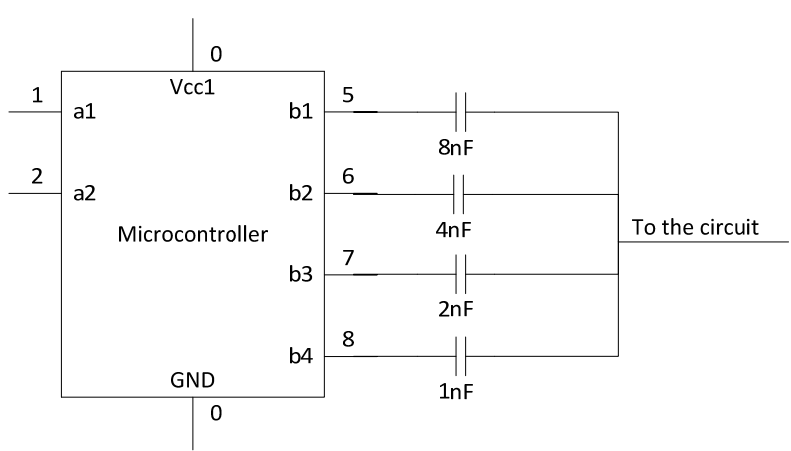

Fig. 3 1-15 nF 4 bit digital capacitor.

$$
2^{3}+2^{2}=8+4=12 \mathrm{nF}
$$

In this paper, the 1-63 $\mathrm{nF} 6$ bit digital capacitor will be used to perform impedance matching.

\subsection{Impedance Matching Algorithm}

Fig. 4 illustrated the circuit model of the RLC band-pass impedance matching circuit. There are two types of impedance used in the calculation, which are source impedance $\left(Z_{s}\right)$, and the channel impedance $\left(Z_{p}\right)$. These variables are measured value in the power line. Therefore, it will vary according to the status of the connected load.

Assuming that $Z_{s}$ and $Z_{p}$ are consisting of real part only, the following method can be used to find $R$ and C:

Let $X=X_{L}-X_{C}$

$$
\begin{gathered}
Z_{s}=j X+\frac{R Z_{p}}{R+Z_{p}}=\frac{j X\left(R+Z_{p}\right)+R Z_{p}}{R+Z_{p}} \\
Z_{p}=\frac{\left(Z_{s}+j X\right) R}{R+Z_{s}+j X}=\frac{R Z s+j X R}{R+Z_{s}+j X}
\end{gathered}
$$

Simplify Eq. (3) to Eq. (5), and Eq. (4) to Eq. (6), therefore:

$$
\begin{aligned}
& R Z_{s}+Z_{s} Z_{p}=R Z_{p}+j X R+j X Z_{p} \\
& R Z_{p}+Z_{s} Z_{p}+j X Z_{p}=R Z_{s}+j X R
\end{aligned}
$$

Let Eq. (5) + Eq. (6),

$$
Z_{s} Z_{p}=j X R
$$

Rearranged Eq. (7) to become:

$$
X=\frac{Z_{s} Z_{p}}{j R}
$$

Substitute Eq. (8) into Eq. (6):

$$
R Z_{p}+Z_{s} Z_{p}+j\left(\frac{Z_{s} Z_{p}}{j R}\right) Z_{p}=R Z_{s}+j\left(\frac{Z_{s} Z_{p}}{j R}\right) R
$$

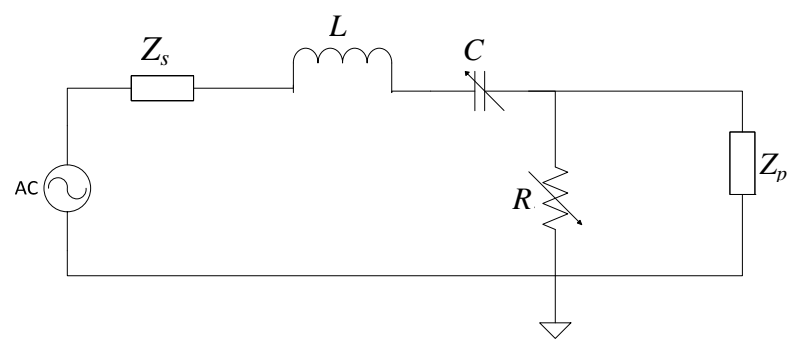

Fig. 4 Proposed RLC band-pass filter impedance matching circuit model.

Rearranged Eq. (9) to become:

$$
R^{2}=\frac{z_{s} z_{p}^{2}}{z_{p}-z_{s}}
$$

The digital capacitor, $C$ can be calculated by $X_{c}=X-X_{L}$, where

$$
C=\frac{1}{2 \pi f X_{c}}
$$

Also, $R$ is obtained from Eq. (10). The value obtained from Eqs. (10) and (11) will compare with the original value of $R$ and $C$, and then adjust the value if it is different from the calculated value. Note that Eqs. (8) and (10) are only valid if $Z_{p}$ is greater than $Z_{s}$, because $R$ has to be positive value all the time.

In order to clarify the error of the calculation, the value obtained from Eqs. (10) and (11) are substituted back into Eqs. (3) and (4) and then the calculated $Z_{s}$ and $Z_{p}$ have been compared with the existing value using Eq. (12), as shown below:

$$
\text { error }=\frac{\left|Z_{\text {measured }}-Z_{\text {calculated }}\right|}{Z_{\text {measured }}} \times 100 \%
$$

By using MATLAB, the computed error is $0 \%$ or a negligible small value. Therefore, this method is applicable to find the value of $R$ and $C$.

\section{Simulated Verification}

In order to verify the feasibility of the concept, the simulation had been performed using MATLAB and PSIM (power simulation software package). The concept had been tested with both minimum load and maximum load. Table 2 tabulated the value of the source impedance and channel impedance during minimum load and maximum load. The impedance value was taken according to the measured value in Ref. 

Narrowband Power Line Communication

[14]. After many trial and error of the simulation, it was decided that $L=5 \mu \mathrm{H}$ inductor was used in this paper to ensure the centre frequency is in the range of $110 \mathrm{kHz}$. The $50 \Omega$ resistor is connected in series with the $Z_{p}$ to prevent the $Z_{p}$ greater than $Z_{s}$.

Table 3 shows the resistance and capacitance value of the circuit, in which they can be calculated using the Eqs. (8), (10) and (11).

Fig. 5 illustrated the circuit diagram of the RLC band-pass filter impedance matching circuit, drawn using PSIM. Meanwhile, Figs. 6 and 7 are the simulated curve for voltages and currents. Note that $V_{\text {in }}$ $=10 \mathrm{~V}$ peak.

From Figs. 6 and 7, the output voltage, $V_{\text {out }}$ and output current, $I_{2}$ are varying depending on the types of load connected to the channel impedance. In Fig. 7, it is clearly shows that the $V_{\text {out }}$ is half of the input voltage, $V_{i n}$, which proves that the maximum power transfer is achieved in the system.
It is crucial to verify whether the modulated frequency signal can pass through the matching circuit. This can be tested using MATLAB. By using the values in Table 3, the magnitude versus frequency curve had been drawn in Figs. 8 and 9 for min and max load. The magnitude of the curve is calculated using the transfer function of the circuit, which is displayed in Eq. (13):

$$
H(j w)=\frac{V_{\text {out }}}{V_{\text {in }}}=\frac{R}{R+j\left(w L-\frac{1}{w C}\right)}
$$

Table 2 Minimum and maximum source and channel impedance.

\begin{tabular}{lll}
\hline \multirow{2}{*}{ Load type } & Source impedance, & Channel impedance, \\
& $Z_{s}(\Omega)$ & $Z_{p}(\Omega)$ \\
\hline Min. load & 15 & $5($ total 55$)$ \\
Max. load & 15 & $250($ total 300$)$ \\
\hline
\end{tabular}

\section{Table 3 Calculated resistance and capacitance value.}

\begin{tabular}{lll}
\hline Load type & Resistance, & Capacitance \\
& $R(\Omega)$ & $C(\mathrm{nF})$ \\
\hline Min. load & 34 & 58 \\
Max. load & 69 & 22 \\
\hline
\end{tabular}

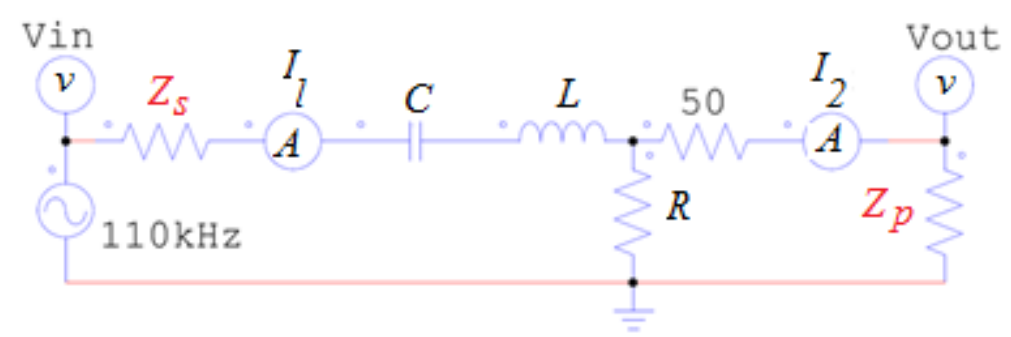

Fig. 5 RLC band-pass filter impedance matching circuit.

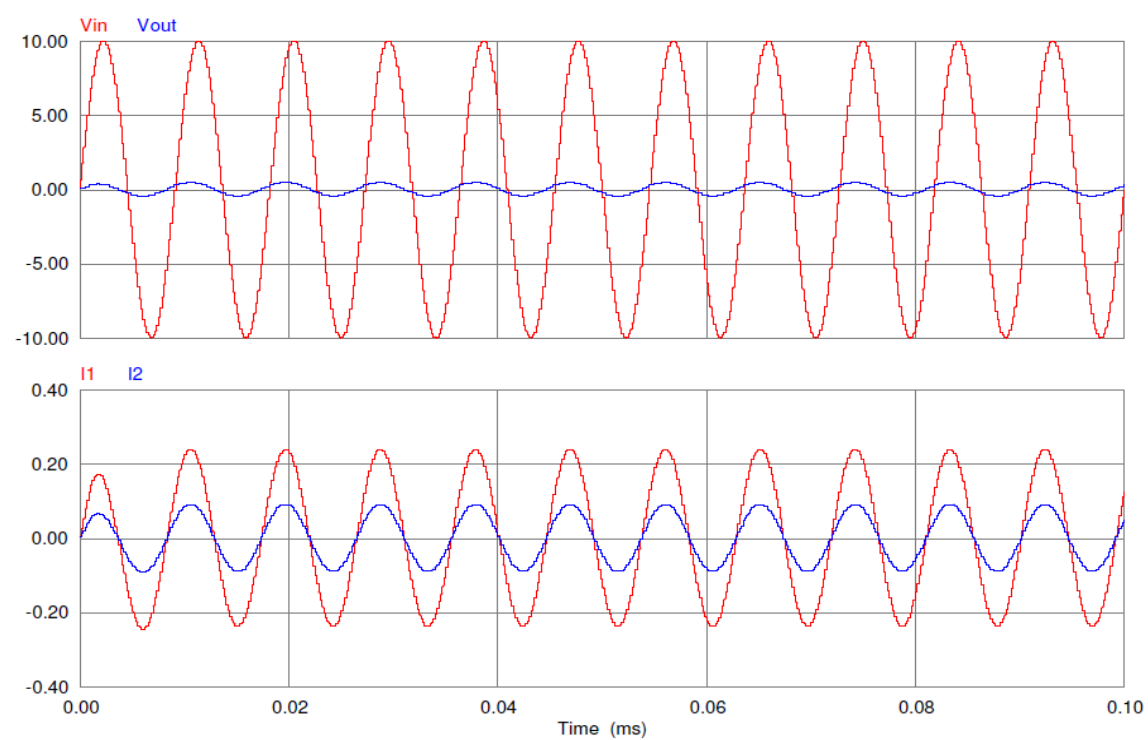

Fig. 6 Voltage and current waveform for min load. 

Narrowband Power Line Communication
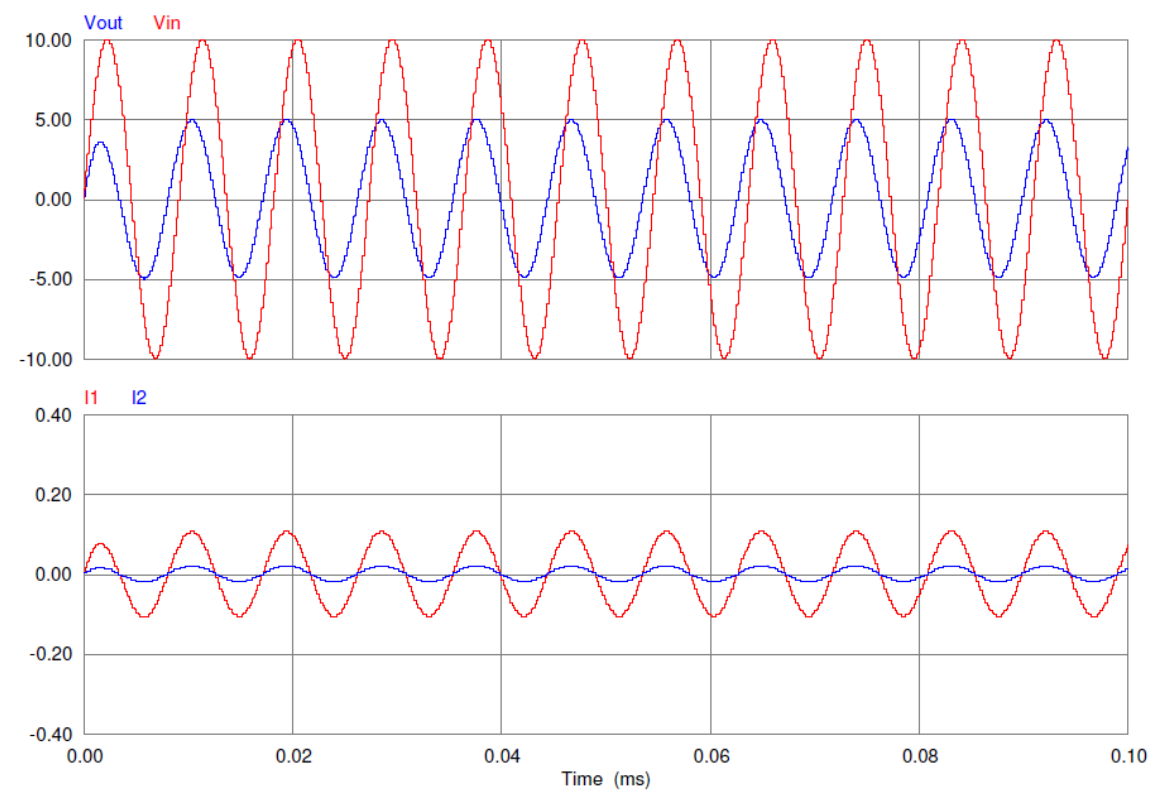

Fig. 7 Voltage and current waveform for max load.

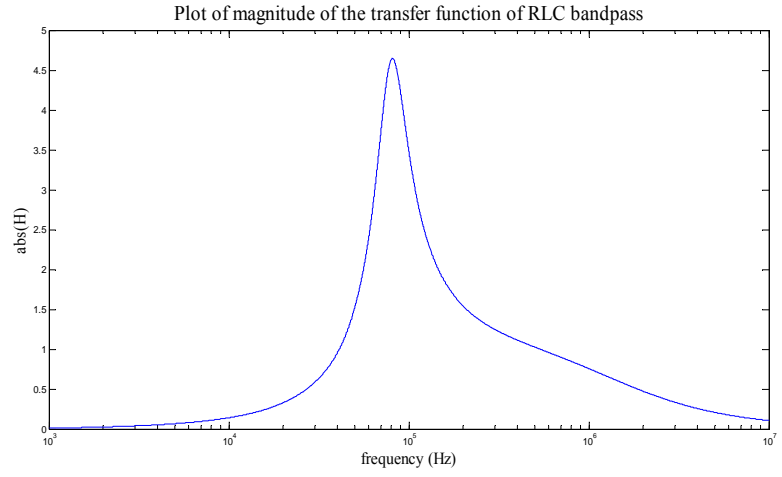

Fig. 8 Magnitude curve of the circuit in min load.

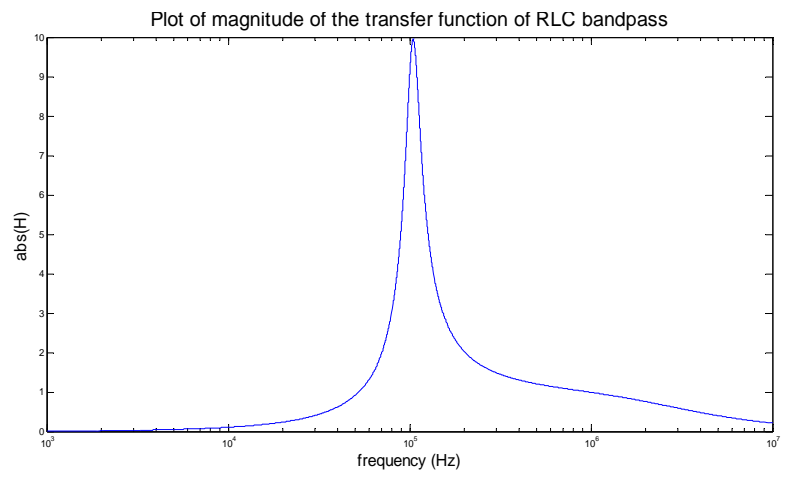

Fig. 9 Magnitude curve of the circuit in max load.

From Figs. 8 and 9, they clearly show that the centre frequency is around $110 \mathrm{kHz}$, and the bandwidth is sufficient for the modulated frequency to pass through. Therefore this impedance matching concept is feasible in the application of PLC.
From the simulated result, it is clearly shows that this methodology is applicable in the NB-PLC channel. Therefore, this impedance matching circuit can be applied in the automation system at home to reduce the noise level and also reduce the data collision rate in the power line communication system. This methodology can also be used in wireless communication system, but different configuration of the components may be required to achieve maximum power transfer in the system. The developed impedance matching circuit is limited to narrowband communication only.

\section{Conclusions}

This paper proposed a new methodology of impedance matching in NB-PLC channel. This method has relatively simple and clear mathematic algorithm to match the PLC source and channel impedances continuously. Therefore, maximum power transfer can be achieved. By comparing with other existing adaptive impedance matching method, this method has advantage in higher matching resolution and easier control. This method had been tested and verified using PSIM and MATLAB, and it is feasible to use in the actual application of NB-PLC, such as home automation systems to reduce noise level and data 
reflection rate. In future, reduction of the power loss and error with the inclusive of reactance in the source and channel impedance will be investigated.

\section{Acknowledgment}

Special thanks to the lecturers and technical staffs in Electrical and Computer Engineering Department, Curtin University Sarawak Malaysia for their help in theoretical analysis, technical guidance and support in publishing this paper.

\section{References}

[1] C.H. Lien, H.C. Chen, Y.W. Bai, M.B. Lin, Power monitoring and control for electric home appliances based on power line communication, in: Proceedings of Instrumentation and Measurement Technology Conference, IMTC 2008, Victoria, 2008, pp. 2179-2184.

[2] C.H. Lien, Y.W. Bai, H.C. Chen, C.H. Hung, Home appliance energy monitoring and controlling based on power line communication, in: ICCE'09, Digest of Technical Papers International Conference on Consumer Electronics, Las Vegas, 2009, pp. 1-2.

[3] L. Lampe, H.C. Ferreira, J. Newbury, T.G. Swart, Power Line Communications: Theory and Applications for Narrowband and Broadband Communications over Power Lines, John Wiley \& Sons, New York, 2010.

[4] D. Shaver, Low Frequency, Narrowband PLC Standards for Smart Grid-The PLC Standards Gap!, Texas Instrument, 2009.

[5] Y. Sun, G. Amaratunga, High-current adaptive impedance matching in narrowband power-line communication systems, in: 2011 IEEE International Symposium on Power Line Communications and Its Applications (ISPLC), Udine, 2011, pp. 329-334.

[6] R.P. Joshi, S. Bhosale, P.H. Patil, Analysis and Simulation of Noise in Power Line Communication Systems, in: 1st International Conference on Emerging Trends in Engineering and Technology, ICETET'08, Nagpur, Maharashtra, 2008, pp. 1287-1292.

[7] M. Katayama, T. Yamazato, H. Okada, A mathematical model of noise in narrowband power line communication systems, IEEE Journal on Selected Areas in Communications 24 (2006) 1267-1276.

[8] R.H. Scott, R. Bullock, Method and system for power line impedance detection and automatic impedance matching, US Patent, 6515485 (2003).

[9] C.Y. Park, K.H. Jung, W.H. Choi, Coupling circuitary for impedance adaptation in power line communications using VCGIC, in: IEEE International Symposium on Power Line Communications and Its Applications, ISPLC 2008, Jeju Island, 2008, pp. 293-298.

[10] M.P. Sibanda, P.A.J. van Rensburg, H.C. Ferreira, Passive, transformerless coupling circuitry for narrow-band power-line communications, in: IEEE International Symposium on Power Line Communications and Its Applications, ISPLC 2009, Dresden, 2009, pp. 125-130.

[11] W.H. Choi, C. Park, A simple line coupler with adaptive impedance matching for power line communication, in: IEEE International Symposium on Power Line Communications and Its Applications, ISPLC'07, Pisa, 2007, pp. 187-191.

[12] Q. Li, J. She, Z. Feng, Adaptive impedance matching in power line communication, in: Proceedings of 4th International Conference on Microwave and Millimeter Wave Technology, Beijing, 2004, pp. 887-890.

[13] D.M. Brown, W.E. Engeler, J.J. Tiemann, High frequency MOS digital capacitor, in: International Electron Devices Meeting, Washington, 1974, pp. 523-526.

[14] H. Tian, R. Yuan, F. Li, Z. Huang, S. Wang, S. Li, et al., Measurement on narrow band power line communication channel impedance of distribution network, in: International Conference on Consumer Electronics, Communications and Networks, Xianning, 2011, pp. 454-457. 